\title{
Cardiac Fiber Inpainting Using Cartan Forms
}

\author{
Emmanuel Piuze ${ }^{1}$, Hervé Lombaert ${ }^{1}$, Jon Sporring ${ }^{1,2}$, and Kaleem Siddiqi ${ }^{1}$ \\ 1 School of Computer Science \& Centre for Intelligent Machines, McGill University \\ 2 eScience Center, Department of Computer Science, University of Copenhagen
}

\begin{abstract}
Recent progress in diffusion imaging has lead to in-vivo acquisitions of fiber orientation data in the beating heart. Current methods are however limited in resolution to a few short-axis slices. For this particular application and others where the diffusion volume is subsampled, partial or even damaged, the reconstruction of a complete volume can be challenging. To address this problem, we present two complementary methods for fiber reconstruction from sparse orientation measurements, both of which derive from second-order properties related to fiber curvature as described by Maurer-Cartan connection forms. The first is an extrinsic partial volume reconstruction method based on principal component analysis of the connection forms and is best put to use when dealing with highly damaged or sparse data. The second is an intrinsic method based on curvilinear interpolation of the connection forms on ellipsoidal shells and is advantageous when more slice data becomes available. Using a database of 8 cardiac rat diffusion tensor images we demonstrate that both methods are able to reconstruct complete volumes to good accuracy and lead to low reconstruction errors.
\end{abstract}

\section{Introduction}

Diffusion Tensor Imaging (DTI) of ex-vivo hearts has been studied extensively across many species, including the human [1], dog [2], goat [3], rat [4] and the pig [5. These studies demonstrate salient and consistent local and global patterns in mammalian cardiac fiber architecture. These patterns are supported by histological measurements [1] and include [6] an inner to outer wall turning of the cardiac fibers in a smooth and regular fashion undergoing a total change of about $110^{\circ}$, and the helical wrapping of fibers around the left ventricle. Such patterns in the left ventricle of the heart relate to its biological function as an efficient pump [7. The study of cardiac fibers thus plays an important role in characterising healthy cardiac function.

As higher resolutions are achieved in ex-vivo diffusion imaging, the contractile nature of cardiac fibers remains to be fully explored. Recent advances in in-vivo DTI have made it possible to measure fiber geometry at different times during a beat cycle. However, current approaches are limited in their resolution, typically providing a few short-axis slices only [8]. In order to generate a complete volume, cardiac fiber directions need to be interpolated from these sparse measurements. Moreover, even with the advent of higher spatial resolutions, (4D) temporal 
imaging could also benefit from a method that can reconstruct intermediate geometry from sparse temporal samples.

Different methods exist for interpolating sparse orientation fields. In [9, a binary mask of the left ventricle is mapped to a prolate spheroid and diffusion tensors are interpolated in the curvilinear coordinates of the surface. Other methods 10 12] carry out PCA on fiber fields and reconstruct fibers by projecting measurements onto the principal components. Diffusion tensors are positive definite symmetric matrices $\in \mathbb{R}^{3 \times 3}$ and their eigenvalues have meaningful physical properties, i.e., they measure water diffusion along three orthogonal directions. The interpolation of diffusion tensors must therefore be done carefully in order to produce physically plausible results, for example using log-euclidean metrics [13.

It is well accepted that in the heart wall the principal direction of diffusion correlates strongly with the underlying cardiac fiber direction [1, and that it varies smoothly in the neighborhood of a voxel, but there is much greater variability in the directions of the second and third eigenvectors. For these reasons we choose to work directly with the principal eigenvector of the diffusion tensor. Motivated by recent work on the differential geometry of heart wall fibers [7, a framework to estimate fiber curvatures was developed in [14] by considering the rotation of a local frame field attached to the fiber direction, using the associated Maurer-Cartan forms. In the present article we extend this approach to reconstruct cardiac fibers from partial measurements.

The methods we develop are based on 1) principal component analysis (PCA) and 2) curvilinear interpolation of the differential structure of a cardiac frame field which is defined from the principal eigenvector of diffusion and from the heart wall normal. By projecting a partial volume onto the differential PCA basis, the differential structure of the frame field can be recovered even when the acquired data is very sparse. For volumes sampled more densely, we also suggest a curvilinear differential interpolation that performs significantly better than the interpolation of the principal eigenvector of diffusion. In Section 2 we describe the natural cardiac coordinate system, the differential descriptors on which we perform PCA and interpolation, and the resulting reconstruction framework. In Section 3 we compare our results with other reconstruction methods. We conclude with a discussion of these results in Section 4.

\section{Methods}

We now discuss the potential of differential geometric studies of cardiac fibers based on the connection forms of [14. This section proposes two methods that employ this approach to interpolate the sparse diffusion data that is typically found in-vivo cardiac diffusion imaging. In Section 2.2, the connection forms are embedded into a partial PCA reconstruction framework. In Section 2.3, an interpolation method based on the natural curvilinear coordinates of the cardiac wall is developed on the connection forms. The experimental results presented in this paper use the publicly available DT-MRI rat dataset of [7. Our computations require a common reference space in order to establish correspondences between 
hearts when comparing the reconstruction results and in computing the PCA basis. An atlas is built using the methods described in [15], and we work with the warped diffusion tensor fields.

\subsection{Measuring Fiber Variation via Connection Forms}

In 14] a framework was introduced to analyze the second-order variability of fiber directions from diffusion data. This framework allows measurements of commonly used cardiac fiber metrics, including the helix and transverse angles, but offers a richer description of the geometry of fiber bundles. This framework requires the existence of a cardiac frame field defined at every voxel in the volume. The principal eigenvector of diffusion can be used to fully define a cardiac orthonormal frame field, which we will refer to as $\boldsymbol{F}_{1}, \boldsymbol{F}_{2}, \boldsymbol{F}_{3} \in \mathbb{R}^{3}$. At a voxel point $\boldsymbol{x}_{0}, \boldsymbol{F}_{1}$ is taken as the direction of the principal eigenvector measured using DT-MRI, $\boldsymbol{F}_{3}$ is the orthogonal component to $\boldsymbol{F}_{1}$ of the gradient $\hat{\boldsymbol{B}}$ of the shortest distance to epicardium i.e. $\boldsymbol{F}_{2}=\left(\hat{\boldsymbol{B}}-\left(\hat{\boldsymbol{B}} \cdot \boldsymbol{F}_{1}\right) \boldsymbol{F}_{1}\right) /\left\|\hat{\boldsymbol{B}}-\left(\hat{\boldsymbol{B}} \cdot \boldsymbol{F}_{1}\right) \boldsymbol{F}_{1}\right\|$ and $\boldsymbol{F}_{2}=\boldsymbol{F}_{3} \times \boldsymbol{F}_{1}$. The local frame $\left[\boldsymbol{F}_{1} \boldsymbol{F}_{2} \boldsymbol{F}_{3}\right]$ is then assembled, with the coordinate axes $\boldsymbol{F}_{1}$ and $\boldsymbol{F}_{2}$ forming a local tangent plane to the heart wall.

Computing Connection Forms. Given a cardiac orthonormal frame field $\boldsymbol{F}_{1}, \boldsymbol{F}_{2}, \boldsymbol{F}_{3} \in \mathbb{R}^{3}$, the action of this frame on the cartesian frame $\boldsymbol{e}_{1}, \boldsymbol{e}_{2}, \boldsymbol{e}_{3}$ is stored in the rotation matrix $\boldsymbol{R}$ where $\left[\begin{array}{lll}\boldsymbol{F}_{1} & \boldsymbol{F}_{2} & \boldsymbol{F}_{3}\end{array}\right]=\boldsymbol{R}\left[\begin{array}{lll}\boldsymbol{e}_{1} & \boldsymbol{e}_{2} & \boldsymbol{e}_{3}\end{array}\right]$. The differential geometry of the frame field is fully characterized by this transformation. $\boldsymbol{R}$ can be used (see e.g. 16] for more details) to express the rate of change of the frame field in an arbitrary direction $\boldsymbol{v}$ in terms of its own coordinate axes:

$$
\left[\begin{array}{c}
\nabla_{v} \boldsymbol{F}_{1} \\
\nabla_{v} \boldsymbol{F}_{2} \\
\nabla_{v} \boldsymbol{F}_{3}
\end{array}\right]=(\mathrm{d} \boldsymbol{R}) \boldsymbol{R}^{-1}\left[\begin{array}{l}
\boldsymbol{F}_{1} \\
\boldsymbol{F}_{2} \\
\boldsymbol{F}_{3}
\end{array}\right]=\boldsymbol{\omega}\left[\begin{array}{c}
\boldsymbol{F}_{1} \\
\boldsymbol{F}_{2} \\
\boldsymbol{F}_{3}
\end{array}\right],
$$

where $\nabla_{v}$ is the differential operator, $\boldsymbol{R}^{-1}=\boldsymbol{R}^{T}$, and $\boldsymbol{\omega}=(d \boldsymbol{R}) \boldsymbol{R}^{-1}$ is a skew symmetric matrix with 3 degrees of freedom formed by the one-forms $\omega_{12}$, $\omega_{13}$, and $\omega_{23}$, called the Maurer-Cartan matrix of connection forms. In order to characterize the change of the principal principal direction of diffusion $\boldsymbol{F}_{1}$ in the direction of the other basis vectors $\boldsymbol{F}_{2}, \boldsymbol{F}_{3}$, we study the six contractions $\omega_{i j k} \equiv \omega_{i j}\left\langle\boldsymbol{F}_{k}\right\rangle$, where $i=1, j \in(2,3)$, and $k \in(1,2,3)$. Each connection form can be computed using

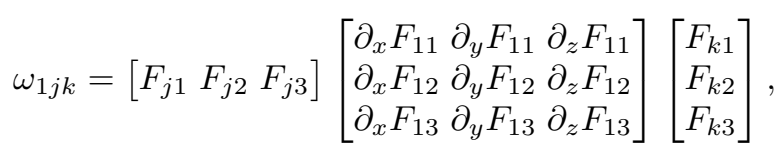

where frame vectors are expressed as $\boldsymbol{F}_{i}=\left(F_{i 1}, F_{i 2}, F_{i 3}\right)$ and partial derivatives are computed using a first order central differentiation scheme. For notational simplicity, we will sometimes label the connection forms $\omega_{p}$ where $p=1, \ldots, 6$, enumerated as $\omega_{121}, \omega_{122}, \omega_{123}, \omega_{131}, \omega_{132}, \omega_{133}$. For the rat dataset of [7], we obtain corresponding values of $0 \pm 0.04,0.01 \pm 0.05,-0.19 \pm 0.13,-0.03 \pm 0.03,0.01 \pm$ $0.03,-0.01 \pm 0.04$, which is in accordance with values reported in [7, 14, 17. 


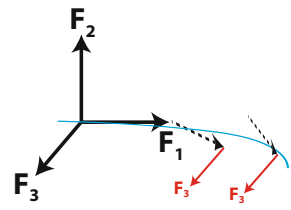

(a) $\omega_{131}$

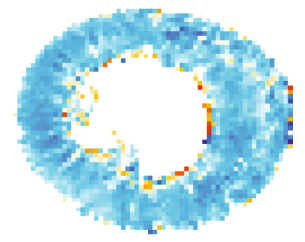

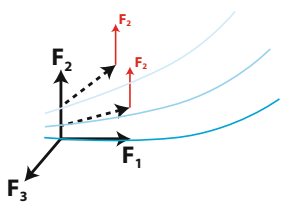

(b) $\omega_{122}$

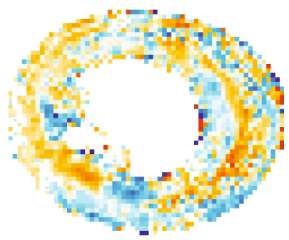

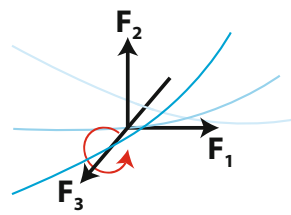

(c) $\omega_{123}$

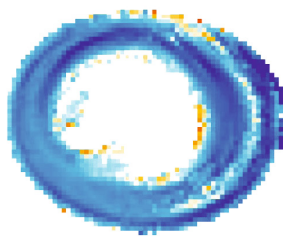

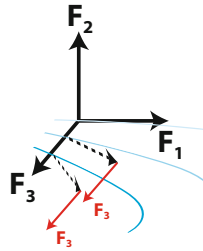

(d) $\omega_{133}$

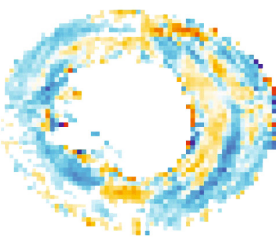

Fig. 1. Connection forms measure the variation of the frames $\boldsymbol{F}_{1}, \boldsymbol{F}_{2}, \boldsymbol{F}_{3}$. Colors range from -0.1 (blue) to 0.1 (red) for $\omega_{131}, \omega_{122}, \omega_{133}$ and -0.5 (blue) to 0.5 (red) for $\omega_{123}$. $\omega_{123}$ and $\omega_{131}$ respectively measure outer to inner wall turning of fibers (the rate of change of the helix angle) and $\omega_{131}$ measures the turning of fibers towards the inner wall (the rate of change of the transverse angle).

Understanding Connection Forms. The connection forms $\omega_{p}$ have a distinctive profile across different hearts. Fig. 1 shows the distribution of a selection of connection forms for the average rat dataset and illustrates their action. The connection form $\omega_{123}$ is particularly smooth across the population while the others can be more disorganized due to the small scale behavior they represent compared to the underlying DTI resolution and noise. Varying degrees of errors are therefore expected in their reconstruction. Each $\omega_{p}$ can be described as follows.

1. The rotations of $\boldsymbol{F}_{1}$ towards $\boldsymbol{F}_{2}, \omega_{12 k}$, describe the manner in which fibers rotate in the tangent plane of the heart. More precisely, $\omega_{121}$ describes geometrical curvature, $\omega_{122}$ describes splaying in the tangent plane, and $\omega_{123}$ describes turning from outer to inner wall. The rotation $\omega_{123}$ measures the rate of change of a salient cardiac feature called the helix angle that has been extensively studied in the cardiac literature.

2. The rotations of $\boldsymbol{F}_{1}$ towards $\boldsymbol{F}_{3}, \omega_{13 k}$, express the turning of the fibers away from the tangent plane. $\omega_{131}$ measures the local topological curvature of the heart, $\omega_{132}$ describes the rate of change of the transverse angle, $\omega_{133}$ measures the fanning of the local fiber population away from the tangent plane and towards the inner wall.

3. The rotations of $\boldsymbol{F}_{2}$ towards $\boldsymbol{F}_{3}, \omega_{23 k}$, are a measure of the variability of the local tangent plane itself. 


\subsection{PCA Reconstruction of Connection Forms}

Following the standard approach to principal component analysis (PCA) and its application to low-dimensional projections [18], we compute PCA on the field of connection forms. The $m$ training measurements $\boldsymbol{\psi}_{p i} \in \mathbb{R}^{n}$ are represented as vectorized matrices, where the subscript $i$ runs over the $n$ voxels contained in the 3D volume, obtained for each $\omega_{p}$ using (2). The mean measurement is defined as $\overline{\boldsymbol{x}}_{p}=\frac{1}{m} \sum_{i} \boldsymbol{\psi}_{p i}$, and is subtracted from each data vector, $\boldsymbol{x}_{p i}=$ $\boldsymbol{\psi}_{p i}-\overline{\boldsymbol{x}}_{p}, \quad i=1, \ldots, m$ to form the data matrix $\boldsymbol{X}_{p}=\left[\boldsymbol{x}_{p 1}, \ldots, \boldsymbol{x}_{p m}\right]$ which is analyzed via PCA. The principal components of each connection form are the eigenvectors of the covariance matrix of $\boldsymbol{X}_{p}, \boldsymbol{C}_{p}=\frac{1}{m} \boldsymbol{X}_{p} \boldsymbol{X}_{p}^{T}$. Given the large number of voxels contained in DT-MRI volumes, it is more efficient to compute the eigenvectors of $\boldsymbol{X}_{p}^{T} \boldsymbol{X}_{p}$. The eigenvectors of $\boldsymbol{C}_{p}$ can then be found as $\boldsymbol{C}_{p} \boldsymbol{w}_{p i}=\lambda_{i} \boldsymbol{w}_{p i}$, where $\boldsymbol{w}_{p i} \equiv \boldsymbol{X} \boldsymbol{v}_{p i}$, and where $\boldsymbol{v}_{p i}$ and $\lambda_{i}$ are respectively the eigenvectors and eigenvalues of $\boldsymbol{X}_{p}^{T} \boldsymbol{X}_{p}$.

Partial volumes can then be projected onto the basis $\left(\boldsymbol{w}_{p i}, \lambda_{i}\right)$. Given a partial volume $\tilde{\boldsymbol{\omega}}_{p}$ of connection forms which is a subset of the complete volume $\boldsymbol{\omega}_{p}$ via a linear map $\tilde{\boldsymbol{\omega}}_{p}=\boldsymbol{L} \boldsymbol{\omega}_{p}$, we want to obtain the partial projection weights $\gamma_{p i}$ onto the PCA eigenbasis that minimizes the following error:

$$
\begin{aligned}
E_{p} & =\left\|\left(\tilde{\boldsymbol{\omega}}_{p}-\overline{\boldsymbol{x}}_{p}\right)-\sum_{i} \gamma_{p i} \boldsymbol{w}_{i}\right\|^{2} \\
& =\left\|\hat{\boldsymbol{\omega}}_{p}-\boldsymbol{\Gamma}_{p} \boldsymbol{W}_{p}\right\|_{2}
\end{aligned}
$$

where $\hat{\boldsymbol{\omega}}_{p}=\tilde{\boldsymbol{\omega}}_{p}-\overline{\boldsymbol{x}}_{p}$ and $\boldsymbol{W}_{p}=\left[\boldsymbol{w}_{p 1}, \ldots, \boldsymbol{w}_{p, m-1}\right]$. The solution is found to be

$$
\boldsymbol{\Gamma}_{p}=\boldsymbol{W}_{p}^{+} \hat{\boldsymbol{\omega}}_{p},
$$

where $\boldsymbol{W}_{p}^{+}$denotes the pseudoinverse of $\boldsymbol{W}_{p}$ and can be obtained using e.g. singular value decomposition. This solution is optimal in the least-squares sense. The resulting partial projection is then found to be

$$
\boldsymbol{\omega}_{p}=\overline{\boldsymbol{x}}_{p}+\boldsymbol{W}_{p}^{+}\left(\tilde{\boldsymbol{\omega}}_{p}-\overline{\boldsymbol{\omega}}_{p}\right) \boldsymbol{W}_{p} .
$$

Using (2) and (6), and denoting $\mathcal{J}_{\tilde{\boldsymbol{F}}_{1}}$ as the Jacobian of $\tilde{\boldsymbol{F}}_{1}$, the following expression is then obtained for projecting a partial cardiac frame measurement $\tilde{\boldsymbol{F}}_{1}, \tilde{\boldsymbol{F}}_{2}, \tilde{\boldsymbol{F}}_{3}$ onto the eigenbasis $\boldsymbol{W}_{p}$ of the connection form $\omega_{1 j k}$ :

$$
\boldsymbol{\omega}_{1 j k}=\overline{\boldsymbol{x}}_{1 j k}+\boldsymbol{W}_{1 j k}^{+}\left(\tilde{\boldsymbol{F}}_{j}^{T} \mathcal{J}_{\tilde{\boldsymbol{F}}_{1}} \tilde{\boldsymbol{F}}_{k}-\overline{\boldsymbol{\omega}}_{1 j k}\right) \boldsymbol{W}_{1 j k} .
$$

\subsection{Reconstruction from Maurer-Cartan Interpolation}

We now introduce the curvilinear radial basis function (RBF) interpolation scheme. Corresponding points in the volume are enforced to lie on a thin shell defined as isolevels of the distance transform to the cardiac outer wall. This 
is supported by observations in 14 that fibers are locally constrained in thin ellipsoidal shells. The interpolation problem can be formulated as follows:

$$
\boldsymbol{\omega}_{p}(\boldsymbol{x})=\sum_{\boldsymbol{x}_{i} \in \zeta} \phi_{i}\left(\boldsymbol{x}, \boldsymbol{x}_{i}\right) \tilde{\boldsymbol{\omega}}_{p}\left(\boldsymbol{x}_{i}\right), \quad \boldsymbol{x}_{i} \in \zeta \cap \tilde{\Omega}, \boldsymbol{x} \in \zeta \cap \Omega, \forall \zeta
$$

where $\boldsymbol{\omega}_{p}(\boldsymbol{x})$ is the connection form volume reconstructed at interpolation points $\boldsymbol{x}$ from the partial measurements $\tilde{\boldsymbol{\omega}}_{p}, \zeta$ is the thin shell domain, $\tilde{\Omega}$ is the interpolation domain, $\boldsymbol{x}_{i}$ are the interpolants contained in the partial measurement domain $\Omega$, and $\phi$ is a radial basis function. We use curvilinear interpolation such that $\phi\left(\boldsymbol{x}, \boldsymbol{x}_{i}\right)=\left|\boldsymbol{x}-\boldsymbol{x}_{i}\right|$. The interpolation is then carried out iteratively for each thin shell $\zeta$, using standard methods for solving the RBF weight matrix [19].

\section{Results}

The temporal and dynamic nature of in-vivo imaging limits the resolution of the DTI volume acquisition and typically only a few short-axis slices are available. We simulate this effect by subsampling the diffusion volumes along the long-axis at regular intervals, as shown in Fig. 2. By varying the number of interval slices, we are able to measure the performance of each method in reconstructing partial diffusion volumes. Equations (7) and (8) offer two complimentary approaches to reconstruct a partial volume of cardiac fiber connection form measurements. These methods are compared against the direct curvilinear interpolation of the eigenvector $\boldsymbol{F}_{1}$ and its PCA reconstruction, followed by connection form measurements using (2). Fig. 2 shows an example of the reconstruction of a $\omega_{123}$ volume consisting of only five short-axis slices. Figure Fig. 3 shows a comparison of the four methods for increasing number of short-axis slices, with the full volume consisting of 50 slices. The connection form interpolation consistently yields lower errors and performs significantly better than both $\boldsymbol{F}_{1}$ - and PCAbased approaches when the volume is more densely sampled. On the other hand, although error curves are comparable for few slices, the interpolation cannot capture the finer details that are better reconstructed by considering extrinsic information content drawn from the population and stored in the PCA basis. The PCA curves stabilize fast, indicating the projection soon converges to a particular weighting of the PCA eigenvectors. More training data in the PCA basis would be required in order to make the reconstruction more subject-specific.

\section{Conclusion}

We proposed two complementary methods for reconstructing partial cardiac fiber volumes using a framework based on the Maurer-Cartan connection forms of the cardiac frame field. The first (extrinsic) method is a partial volume reconstruction using a PCA of connection forms. This method is more appropriate when facing highly sparse and irregular data. One limitation of this approach is in the number of sample points required in constructing the PCA basis. Given 


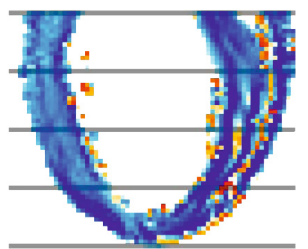

(a) Original

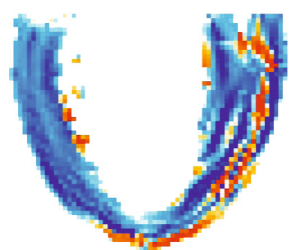

(b) $\boldsymbol{F}_{1}$ interpolation

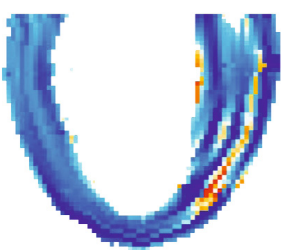

(c) $\omega$-interpolation

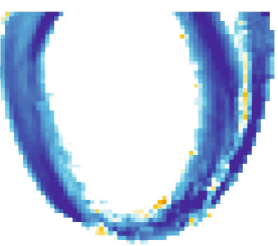

(d) $\omega-\mathrm{PCA}$

Fig. 2. Reconstruction of the connection form $\omega_{123}$ for one specimen. From left to right: original $\omega_{123}$ volume and axial subsamples (overlaid in gray), $\boldsymbol{F}_{1}$ interpolation method, connection form interpolation method, connection form PCA method. $\omega_{123}$ has average magnitude 0.2376 and the total errors are respectively $0.1446,0.1063,0.1275$.

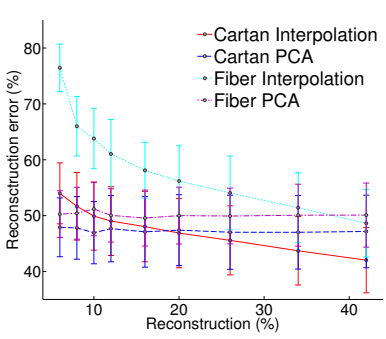

(a) $\omega_{131}$

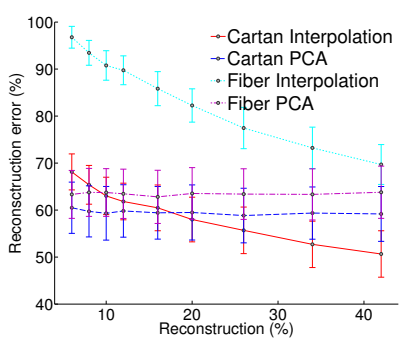

(b) $\omega_{122}$

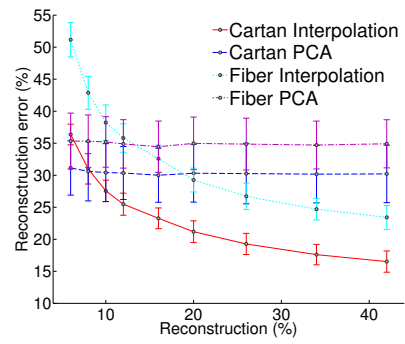

(c) $\omega_{123}$

Fig. 3. Reconstruction error of three selected connection forms using different interpolation schemes. Proposed methods are shown in red and blue.

the stability of the cardiac frame field across different subjects, collecting more exemplar data would result in a PCA basis that captures a larger amount of the variability found in the population and consequently provide a more robust reconstruction. Another drawback of this method is that interpolants are not reconstructed exactly due to the projection step of the PCA. The second (intrinsic) method is a ellipsoidal curvilinear interpolation of the connection forms following thin shells of the cardiac wall. This method yields lower errors as more reconstruction slices are available and goes exactly through interpolants. One drawback of using RBF interpolation is that basis functions become less meaningful if interpolants are spatially distant, which can happen with highly sparse diffusion volumes. Moreover, salient small-scale patterns located in-between interpolants cannot be reconstructed without a priori information. In that case the problem would be better expressed via PCA reconstruction.

There are a number of directions for future investigation including the development of partial differential equation solvers for reconstructing frame fields from connection form measurements, the use of connection forms for temporal interpolation and superresolution of diffusion volumes, and the combination of intrinsic and extrinsic data into a united PCA-interpolation framework.

Acknowledgments. We thank NSERC and FQRNT for research funding. 


\section{References}

1. Rohmer, D., Sitek, A., Gullberg, G.T.: Reconstruction and visualization of fiber and laminar structure in the normal human heart from ex vivo diffusion tensor magnetic resonance imaging (dtmri) data. Invest. Radiol. 42(11), 777-789 (2007)

2. Helm, P.A., Tseng, H.J.J., Younes, L., McVeigh, E.R., Winslow, R.L.: Ex vivo $3 \mathrm{D}$ diffusion tensor imaging and quantification of cardiac laminar structure. MRM 54(4) (2005)

3. Geerts, L., Bovendeerd, P., Nicolay, K., Arts, T.: Characterization of the normal cardiac myofiber field in goat measured with MR-diffusion tensor imaging. AJP: Heart and Circ. Physiol. 283(1) (2002)

4. Bishop, M.J., Hales, P., Plank, G., Gavaghan, D.J., Scheider, J., Grau, V.: Comparison of rule-based and DTMRI-derived fibre architecture in a whole rat ventricular computational model. In: Ayache, N., Delingette, H., Sermesant, M. (eds.) FIMH 2009. LNCS, vol. 5528, pp. 87-96. Springer, Heidelberg (2009)

5. Zhang, L., Chen, J., Gibson, A., Holland, M., Lanza, G., Wickline, S.: 110 myofiber developmental plasticity in fetal hearts delineated with diffusion tensor MRI. CMR $10(2008)$

6. Streeter, D.D.: Gross morphology and fiber geometry of the heart. In: Handbook of Physiology, Section 2. The Heart, pp. 61-112. Williams and Wilkins (1979)

7. Savadjiev, P., Strijkers, G.J., Bakermans, A.J., Piuze, E., Zucker, S.W., Siddiqi, K.: Heart wall myofibers are arranged in minimal surfaces to optimize organ function. Proc. Natl. Acad. Sci. USA 109(24), 9248-9253 (2012)

8. Mekkaoui, C., Nielles-Vallespin, S., Gatehouse, P.D., Jackowski, M.P., Firmin, D.N., Sosnovik, D.E.: Diffusion mri tractography of the human heart in vivo at end-diastole and end-systole. CMR 14, 1-2 (2012)

9. Toussaint, N., Sermesant, M., Stoeck, C.T., Kozerke, S., Batchelor, P.G.: In vivo human 3D cardiac fibre architecture: Reconstruction using curvilinear interpolation of diffusion tensor images. In: Jiang, T., Navab, N., Pluim, J.P.W., Viergever, M.A. (eds.) MICCAI 2010, Part I. LNCS, vol. 6361, pp. 418-425. Springer, Heidelberg (2010)

10. Merchant, S., Jiang, Y., Joshi, S., Hsu, E.: Assessment of variability of the mouse myocardial fiber structure via principal component analysis. In: WorldComp (2012)

11. Gil, D., Garcia-Barnes, J., Hernández-Sabate, A., Marti, E.: Manifold parametrization of the left ventricle for a statistical modelling of its complete anatomy. In: SPIE (2010)

12. Lekadir, K., Ghafaryasl, B., Muñoz-Moreno, E., Butakoff, C., Hoogendoorn, C., Frangi, A.F.: Predictive modeling of cardiac fiber orientation using the Knutsson mapping. In: Fichtinger, G., Martel, A., Peters, T. (eds.) MICCAI 2011, Part II. LNCS, vol. 6892, pp. 50-57. Springer, Heidelberg (2011)

13. Arsigny, V., Fillard, P., Pennec, X., Ayache, N.: Fast and simple calculus on tensors in the log-euclidean framework. In: Duncan, J.S., Gerig, G. (eds.) MICCAI 2005. LNCS, vol. 3749, pp. 115-122. Springer, Heidelberg (2005)

14. Piuze, E., Sporring, J., Siddiqi, K.: Moving frames for heart fiber geometry. In: Gee, J.C., Joshi, S., Pohl, K.M., Wells, W.M., Zöllei, L. (eds.) IPMI 2013. LNCS, vol. 7917, pp. 524-535. Springer, Heidelberg (2013)

15. Lombaert, H., Peyrat, J.M., Croisille, P., Rapacchi, S., Fanton, L., Cheriet, F., Clarysse, P., Magnin, I., Delingette, H., Ayache, N.: Human atlas of the cardiac fiber architecture: Study on a healthy population. TMI 31 (2012) 
16. Koenderink, J.: Solid shape, vol. 2. Cambridge Univ. Press (1990)

17. Piuze, E., Lombaert, H., Sporring, J., Strijkers, G.J., Bakermans, A.J., Siddiqi, K.: Atlases of cardiac fiber differential geometry. In: Ourselin, S., Rueckert, D., Smith, N. (eds.) FIMH 2013. LNCS, vol. 7945, pp. 442-449. Springer, Heidelberg (2013)

18. Crimi, A., Lillholm, M., Nielsen, M., Ghosh, A., de Bruijne, M., Dam, E.B., Sporring, J.: Maximum a posteriori estimation of linear shape variation with application to vertebra and cartilage modeling. TMI 30(8), 1514-1526 (2011)

19. Buhmann, M.D.: Radial basis functions: theory and implementations, vol. 12. Cambridge University Press (2003) 